\title{
Fabrication of a Thermosensitive In Situ Gel Nanoemulsion for Nose to Brain Delivery of Temozolomide
}

\author{
Masoumeh Bayanati $\left(\mathbb{D},{ }^{1}\right.$ Abolfazl Ghafouri Khosroshahi $\left(\mathbb{D},{ }^{2}\right.$ Maryam Alvandi $\left(\mathbb{D},{ }^{3}\right.$ \\ and Mohammad Mehdi Mahboobian (iD) ${ }^{1}$ \\ ${ }^{1}$ Department of Pharmaceutics, School of Pharmacy, Hamadan University of Medical Sciences, Hamadan, Iran \\ ${ }^{2}$ Department of Medicinal Chemistry and Nuclear Medicine, School of Pharmacy, Farshchian Cardiovascular Subspecialty \\ Medical Center, Hamadan University of Medical Sciences, Hamadan, Iran \\ ${ }^{3}$ Department of Nuclear Medicine, School of Medicine, Hamadan University of Medical Sciences, Hamadan, Iran
}

Correspondence should be addressed to Mohammad Mehdi Mahboobian; m.mahboobian@umsha.ac.ir

Received 21 May 2020; Revised 29 January 2021; Accepted 25 February 2021; Published 13 March 2021

Academic Editor: Fengqiang Sun

Copyright (c) 2021 Masoumeh Bayanati et al. This is an open access article distributed under the Creative Commons Attribution License, which permits unrestricted use, distribution, and reproduction in any medium, provided the original work is properly cited.

In this study, a thermosensitive in situ gel nanoemulsion was formulated by a low energy method for intranasal delivery of temozolomide to bypass the blood-brain barrier and optimize chemotherapy for glioblastoma. Various amounts of Labrasol, Transcutol ${ }^{\circledR} \mathrm{P}$, and Triacetin were chosen as nanoemulsion components based on the solubility and the partial pseudoternary phase diagrams studies. Poloxamer derivatives added to the selected nanoemulsion and gelling temperature optimized. The prepared in situ gel nanoemulsion containing temozolomide showed a mean droplet size of $16.25 \pm 0.44 \mathrm{~nm}$, a polydispersity index value of $0.35 \pm 0.01$, and desirable $\mathrm{pH}$ and viscosity. In vitro release studies revealed that both nanoemulsion and in situ gel preparation have sustained release pattern in comparison to the control solution. Visual evaluation and droplet size and polydispersity index measurements showed both nanoemulsion and in situ gel nanoemulsion were stable during heating-cooling and freeze-thaw cycles and also centrifugation. Mucoadhesion percentage of in situ gel nanoemulsion was $37.037 \pm 2.32$ regarding ex vivo studies, which had a significant rise in comparison to control solution and nanoemulsion. Permeation across the nasal mucosa was 1.43- and 1.52-fold higher than the control solution for nanoemulsion and in situ nanoemulsion, respectively. Gamma scintigraphy study showed brain accumulation of developed nanoemulsion formulations. Our studies demonstrated optimized formulation has suitable physicochemical properties, desirable release profile, enhanced permeation across the nasal mucosa, and prolonged resistance time at the nasal mucosa. Therefore, in situ gel nanoemulsion would be an effective novel nasal delivery system for the treatment of glioblastoma.

\section{Introduction}

Brain tumor treatment is one of the most challenging issues of central nervous system disease [1]. About 238,000 diagnoses of the brain and other central nervous system tumors occur every year, all around the world. Based on WHO (world health organization) classification, gliomas refer to the primary brain tumors with the origin of glial cells, which include nearly $80 \%$ of malignant brain tumors [2]. Grade IV gliomas, glioblastoma multiforme (GBM), is the most prevalent and aggressive type of primary brain tumors with an incidence of 5-8 per 100,000 population [3]. Currently, stan- dard therapy for glioma is resection of tumors by surgical as much as possible and chemotherapy along with radiotherapy and/or photodynamic therapy $[4,5]$. But despite available treatments, GBM's prognosis is still poor, and median survival from diagnosis is not more than 15 months [6]. So, it is essential to optimize every concept of treatment, especially chemotherapy. Failure in chemotherapy in this type of tumors is not related to the poor chemotherapy activity of existing agents, but to the presence of the protective microvasculature of the central nervous system (CNS) named the blood-brain barrier (BBB) [1]. Temozolomide (TMZ, Temodar $\left.^{\circledR}\right)$, the first line antineoplastic agent for the treatment of 
GBM, is FDA approved orally and intravenously [7, 8]. Even though this second-generation DNA-alkylating agent is permeable to the BBB, it must be administrated in high doses in systemic use due to its short serum half-life [7, 9]. But this high-dose TMZ can lead to an increased risk of a variety of adverse effects such as hematological toxicity and cardiomyopathy [10].

Recently, new technologies such as implantable controlled release systems, convection-enhanced delivery (CED), and nanoparticles have created this opportunity to deliver drugs to the CNS without crossing the BBB and so promising this approach to select agents for the treatment of brain tumors mostly based on their ability for destroying tumors and not regarding their potential of crossing the $\mathrm{BBB}$ [11]. In recent decades, using the nasal cavity has also gained prominence for bypassing the BBB. Administration drug through the nasal cavity where olfactory and trigeminal nerve terminations provide the sole connection between the CNS and the environment, therefore, seems to be efficient for direct drug delivery to the brain. This strategy is noninvasive, painless, highly safe, remarkably easy to use and represents a rapid onset of action as well as minimizing systemic exposure, and all these advantages increase patient compliance. It also bypasses hepatic first-pass metabolism, which decreases the therapeutic dose [12]. Despite remarkable advantages, nasal drug delivery has limitations, including degradation of some drugs at the mucosal surface, presence of clearance systems in the nasal cavity, and also possible mucosal irritation and/or damage or even allergies $[12,13]$. The half-life of the clearance for both powder and liquid in the nasal cavity is $15-20$ minutes. And the applied volume of the formulation is limited to $25-200 \mu \mathrm{l}$ each time [14]. These restrictions must be considered in formulation design.

Lipidic systems like nanoemulsions (NEs) are favorable for nasal drug delivery, primarily because of the biocompatibility reasons [13]. NEs are novel drug delivery systems that can be efficiently applied in nasal dosage forms. Low energy or high energy methods may be used to prepare oil in water or water in oil dispersions stabilized in the presence of appropriate surfactant(s). Using NEs, problems relating to solubility and stability including oxidation, $\mathrm{pH}$ hydrolysis, and enzymatic degradation can be managed [12]. But formulation may be washed out by the nose clearance system before desirable absorption occurs. The prolonged resistance time of the formulation in the nasal cavity results in an extended release of drugs from the nanodroplets. The possible efficient approaches to prolong drug resistance time at the nasal mucosa are enhancing viscosity or applying gelling systems. In situ gel systems are pharmaceutical dosage forms that are liquid in shelf-condition and convert to the gel after application at the site of the applied responding to $\mathrm{pH}$, temperature, or ionic changes. While having all conventional gels advantages, in situ gels can be easily instilled and provide a reproducible dosing [15].

Among all in situ gelling systems, thermosensitive ones are more favorable in drug delivery as upon administration, the temperature rises spontaneously, and phase transition occurs [16]. To develop thermosensitive in situ gel formulations for nasal use, the transition temperature must be set in the range of $25-37^{\circ} \mathrm{C}$ [17]. The most common mucoadhesive copolymer using in these formulations is poloxamer 407 , which undergoes micellization and gelation as increasing temperature [18]. Poloxamer 407 is rarely used alone because of its low gelling temperature, and other poloxamer copolymers are added to the formulation to modify the gelling temperature [19].

Insignificant toxicity, suitable drug release properties, compatibility with numerous chemical agents, nonirritating action on the mucosal surface, prolonged resistance time at a specific drug delivery site, decreasing the therapeutic dosage, and the side effects investigate poloxamer 407 as a suitable gelling agent [20-22]. Poloxamer can develop micelles; it improves solubilization of poorly water-soluble drugs; therefore, sufficient amounts of these drugs can be dissolved in a small volume of the formulation, which is suitable for intranasal use [23]. Poloxamer also increases drug permeation through the mucosal surface, probably because it is a nonionic surfactant and reduces viscosity and elasticity of the mucosa and also perturbs lipid membrane and improves lipid leakage through it $[24,25]$.

Even though the potential toxicity of the developing formulations for intranasal use is concerning, previous studies have demonstrated any adverse effect of prepared formulations containing TMZ on $16 \mathrm{HBE}$ cell line and porcine nasal mucosa as standard models of human nasal mucosa cells and tissues $[3,26]$. Furthermore, NEs as drug vehicles for brain targeted drugs were found to be safe upon administration via nasal route [27-29]. Histological evaluations on developed nimodipine mucoadhesive microemulsion consist of labrasol, Transcutol ${ }^{\circledR} \mathrm{P}$, poloxamer 407 , and poloxamer 188 as excipients showed any damage on rat nasal mucosa [30]. In a recent study, teriflunomide loaded NE with similar constituents, despite having anticancer activity was found to be safe for normal cells. This formulation had desirable biodistribution upon nasal administration on Wistar rats and less accumulated in the kidney and liver, without any necrosis or damage on the nasal mucosa [31].

In this study, we developed and characterized a thermosensitive in situ gel NE for intranasal administration of TMZ to overcome nasal clearance systems, prolong the formulation resistance time at the nasal mucosa, improve drug absorption, bypass the $\mathrm{BBB}$, and as a result optimize drug delivery of TMZ.

\section{Materials and Methods}

2.1. Materials. The following materials were used in the study:

TMZ powder was purchased from Senova Technology. CO, China. Poloxamer407, Poloxamer188, and dialysis tubing cellulose membrane were supplied from Sigma Aldrich Chemical Company, USA. Triacetin was provided by Samchun Chemical Co., Ltd, South Korea. Labrasol and Transcutol ${ }^{\circledR} \mathrm{P}$ were obtained as gift samples from Gattefosse, France. All other chemicals and reagents were of analytical grade. 


\subsection{Methods}

2.2.1. Solubility Studies. Excess amount of TMZ was added to $1 \mathrm{ml}$ of various oils (Oleic acid, Triacetin, Capryol 90), Cosurfactants (Propylene glycol, Transcutol ${ }^{\circledR} \mathrm{P}$, polyethylene glycol 400), and 15\% surfactants aqueous solutions (Tween 80 , Labrasol, Cremophor ${ }^{\circledR} \mathrm{RH} 40$ ) separately to determine the solubility of the drug. The mixtures were allowed to reach equilibration in a shaker incubator at $200 \mathrm{rpm}$ for 24 hours at $25^{\circ} \mathrm{C}$. Then, samples were centrifuged at $13000 \mathrm{rpm}$ for 10 minutes. The supernatants were separated and diluted with methanol. Then, UV spectrophotometry quantification at $327 \mathrm{~nm}$ represented TMZ concentration.

\subsubsection{Construction of Partial Pseudoternary Phase Diagrams.} The selected oil, surfactant, and cosurfactant based on solubility studies were used to develop NEs and construct partial pseudoternary phase diagrams by the water titration method. Labrasol as a surfactant and Transcutol ${ }^{\circledR} \mathrm{P}$ as a cosurfactant were mixed in various mass ratios $(2: 1,1: 1,1: 2)$ to obtain surfactant mixture $\left(S_{\text {mix }}\right)$. These mixtures were blended with different weight ratios of triacetin as an oil (from 9:1 to $40: 60(w / w))$ in screw-cap glass vials separately. Then, purified water was added to each mixture dropwise while stirring at room temperature. After adding each volume of water and equilibration, samples were monitored visually, and their clarity state was reported as transparent, semitransparent, or turbid. At the end of the observations, the software sigma-plot ver.12 was applied to construct a pseudoternary phase diagram for each $S_{\text {mix }}$ separately and determine the NE regions.

2.2.3. Preparation of NEs Containing TMZ. According to the phase diagrams, the suitable ratios of surfactant, cosurfactant, and oil were selected to fabricate nanoemulsions. NEs were prepared by a low energy method in which the intended quantity of surfactant, cosurfactant, oil, and also $0.15 \% \mathrm{w} / \mathrm{w}$ TMZ were weighted and blended at gentle heat while stirring to obtain a transparent mixture [32]. Then, the calculated amount of purified water was added to the mixture gradually, and the whole formulation was stirred at room temperature at least for an hour to prepare clear homogenous NEs.

\subsubsection{Physicochemical Characterization of Formulations} Containing TMZ. The following methods have been utilized to measure physicochemical properties of preparations.

Droplet size, polydispersity index, and zeta potential were measured immediately after preparation using a Malvern Zetasizer NanoZS (Malvern, United Kingdom). pH meter (Sartorius, Switzerland) was used to evaluate the $\mathrm{pH}$ values of formulations at room temperature. This $\mathrm{pH}$ meter was three-point calibrated ( $\mathrm{pH} 4, \mathrm{pH} 7$, and $\mathrm{pH} 10)$ with standard buffer solutions to ensure instrument validity. Brookfield DVII viscometer (Brookfield Engineering Laboratories Inc., USA) was applied to determine the rheological properties of the samples at room temperature with a speed rate from 50 to $200 \mathrm{rpm}$. Spindle CP-34 was utilized in this measurement. All measurements were carried out in triplicate, and the results are declared as average value \pm standard deviation.
2.2.5. Preparation of In Situ Gel NEs. To obtain in situ gel NE with an optimum gelling temperature, poloxamer 407 and poloxamer 188 in different weight ratios were weighed and dissolved in the aqueous phase of the selected formulation (regarding physicochemical characterization) by stirring at $4^{\circ} \mathrm{C}$ overnight [33]. Then, this aqueous phase was added drop by drop to the oily phase prepared as described in NE preparation, and the sample was stirred at $4^{\circ} \mathrm{C}$ at least for an hour to achieve a homogenous formulation. The prepared formulations were kept in the refrigerator until performing further studies.

2.2.6. Determination of Gelation Temperature of In Situ Gel NEs. The gelation temperature of each formulation was determined by pouring $250 \mu \mathrm{l}$ of each formulation in the vials and then placing it in a water bath (Memmert ONE10, Germany) with a temperature of $15^{\circ} \mathrm{C}$. This initial temperature was increased gradually (at a rate of $1^{\circ} \mathrm{C} / \mathrm{min}$ ). The vials were rotated at $180^{\circ}$ angle each minute, and at the temperature that phase transition had been occurring, the formulation could not flow, which was reported as gelation temperature [34]. The formulation with gelation temperature near the nose cavity temperature $\left(32-34^{\circ} \mathrm{C}\right)$ was selected as the optimum one [35].

2.2.7. In Vitro Drug Release Studies. The drug release from developed TMZ-loaded NE, in situ gel NE, and aqueous solution as control was evaluated by the dialysis bag method. The dialysis bag (MW cut-off $12400 \mathrm{Da}$ ) was presoaked in purified water and kept in the refrigerator for 24 hours. $1 \mathrm{ml}$ of each sample was placed whiten a dialysis bag separately, and both ends of the bags were sealed.

Each bag punched to a paddle of USP dissolution apparatus type II and floated in $250 \mathrm{ml}$ of phosphate-buffered saline (PBS, $\mathrm{pH}=6.4$ ), with a rotation speed of $50 \mathrm{rpm}$ for $6 \mathrm{~h}$ at $34 \pm 1^{\circ} \mathrm{C}$. At the defined times $(15,30,45,60,90,120,180$, 240,300 , and $360 \mathrm{~min}$ ), $2 \mathrm{ml}$ of release medium was withdrawn and replaced with fresh PBS solution immediately. To determine the amount of TMZ in releasing medium at each of these particular times, we measured the concentration of drug in the sample medium by using UV spectrophotometry at $327 \mathrm{~nm}$.

2.2.8. Accelerated Physical Stability Studies. The stability of the selected NE and in situ gel NE was investigated by accomplishing six heating-cooling cycles $\left(4^{\circ} \mathrm{C}\right.$ and $\left.40^{\circ} \mathrm{C}\right)$, three freeze-thaw cycles $\left(-21^{\circ} \mathrm{C}\right.$ and $\left.25^{\circ} \mathrm{C}\right)$, and centrifugation studies (13000 rpm for $30 \mathrm{~min}$ ).

After performing each test, samples were monitored visually for any possible instability phenomena, including phase separation, clarity changes, creaming, flocculation, cracking or coalescence, and ext. Also, the droplet size and the polydispersity index were measured before and after the exam [3638].

2.2.9. Ex Vivo Mucoadhesion Study. Modified falling liquid film method performed to evaluate the mucoadhesive properties of in situ gel NE, NE, and an aqueous solution containing $0.15 \% w / w$ TMZ (as control). Bovine nasal mucosa was collected from a local slaughterhouse. PBS 


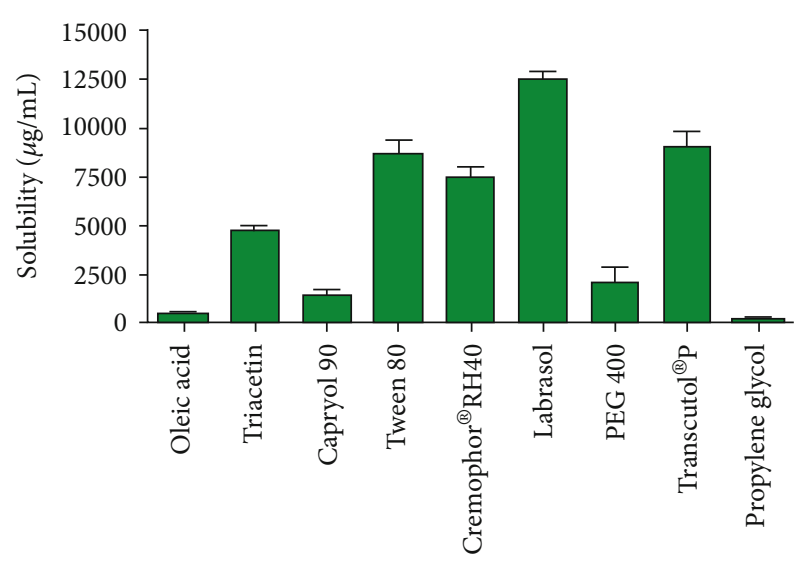

FIGURE 1: Graphic representation of solubility of TMZ in various oils (Oleic acid, Triacetin and Capryol 90), surfactants (Tween 80, Cremophor ${ }^{\circledR} \mathrm{RH} 40$ and Labrasol), and cosurfactants (PEG 400, Transcutol ${ }^{\circledR} \mathrm{P}$ and Propylene glycol). Each value represents mean $\pm \mathrm{SD}(n=3)$.

solution was used to wash mucosa to ensure there is no adhered tissue to the mucosal surface. Then, nasal mucosa was cut into $5 \mathrm{~cm}$ long rectangle shape pieces. A piece attached to a plastic tube that was cut into a circular shape and held at an angle of $90^{\circ} \mathrm{C}$ and then 250 of one formulation (containing $0.375 \mathrm{mg}$ of TMZ) was placed on the mucosa and incubated for 5 minutes in the exposure of a fairly warm air storm simulating nasal cavity condition. Then, the angel was changed to $45^{\circ}$, and a peristaltic pump used to pump PBS ( $\mathrm{pH} 6.4$ and temperature $34^{\circ} \mathrm{C}$ ) over the nasal mucosa at the rate of $5 \mathrm{ml} / \mathrm{min}$. The washed fluid was collected over $5 \mathrm{~min}$ continuously. The amount of TMZ in this fluid represented the nonadhered sample, which was measured spectrophotometrically at $327 \mathrm{~nm}$. The difference between the amount of nonadhered drug and the amount of the applied drug was estimated as the adhered drug amount. The ratio between adhered and the applied drug amount was determined as mucoadhesion percentage $[39,40]$.

2.2.10. Ex Vivo Drug Permeation Studies. Bovine nasal mucosa is collected from a local slaughterhouse in PBS. After washing adhered tissues on the mucosal surface by PBS, the excised mucosal tissues were placed between the donor and receptor compartment of a Frantz diffusion cell. The donor compartment with $17 \mathrm{~mm}$ orifice diameter displayed a permeation area of $2.27 \mathrm{~cm}^{2}$. The receptor compartment with a volume of $12 \mathrm{ml}$ filled with buffer phosphate $(\mathrm{pH}=6.4)$ and the temperature during the test performing was kept at $34 \pm 1^{\circ} \mathrm{C}$. The sufficient volume of each sample (aqueous solution as a control, NE, in situ gel $\mathrm{NE}$ ) equivalent to $1 \mathrm{mg}$ of TMZ was placed in the donor compartment on the outer surface of the nasal mucosa. The permeation study was carried out for 6 hours. At the defined times $(30,60,90,120,180,240$, 300 , and $360 \mathrm{~min}$ ), $0.5 \mathrm{ml}$ of the aliquots were withdrawn and analyzed for measuring the amount of permeated drug by using UV spectrophotometry method at $327 \mathrm{~nm}$.
2.2.11. UV Analysis of TMZ. The determination of the TMZ was done by validated UV spectrophotometry method. Briefly, a stoke solution of TMZ $(1 \mathrm{mg} / \mathrm{ml})$ was procured by dissolving a precisely measured amount of TMZ in methanol; then, the standard working solutions were obtained by a series of dilutions with buffer phosphate solution $(\mathrm{pH}=6.4$ ). The absorbance rate of the prepared solutions was determined at $327 \mathrm{~nm}$ by SPECORD $210 \mathrm{UV}$ spectrophotometer (Analytik, Germany). The developed method displayed linear response $\left(y=0.0493 x-0.00039, r^{2}=0.999\right)$ in the concentration range of 1.56 to $25 \mu \mathrm{g} / \mathrm{ml}$ of TMZ with LOD and LOQ of 117 and $354 \mathrm{ng} / \mathrm{ml}$, respectively.

2.2.12. Gamma Scintigraphy. Developed TMZ-loaded NEs was radiolabeled with technetium $(99 \mathrm{mTc})$ by direct labeling method in the radiopharmacy lab of the Department of Nuclear Medicine in Hamadan University.

In this method, initially, the pertechnetate ( $\mathrm{TcO} 4-)$ was reduced with tin chloride dehydrate $(\mathrm{SnCl} 2 \bullet 2 \mathrm{H} 2 \mathrm{O})$ in order to radiolabel TMZ. To reduce the $99 \mathrm{mTc}, 15 \mu \mathrm{g}$ tin chloride dehydrate $(\mathrm{SnCl} 2.2 \mathrm{H} 2 \mathrm{O})$ was added into $2 \mathrm{mCi}$ pertechnetate. Using sodium bicarbonate, the $\mathrm{pH}$ of the solution was adjusted to $\approx 6.5-7$.

Aqueous solution of $99 \mathrm{mTc}(2 \mathrm{mCi})$ was mixed with the formulations and incubated for $15 \mathrm{~min}$. The radiolabeling efficiency was examined by thin layer chromatography using acetone as a mobile phase. After confirmation of radiolabeling process, Wistar rats weighted 250-275 g was anesthetized using (peritoneal injection of ketamine $(100 \mathrm{mg} / \mathrm{ml}$ ) and $25 \mu \mathrm{l}$ of the radiolabeled formulation was administrated intranasally to each nostril. Then, the rats were placed on the imaging platform. The images were recorded at selected time of 0,15 , and $30 \mathrm{~min}$ postadministration by Symbia Evo Excel, Siemens Healthcare.

2.2.13. Statistical Analysis. All experiments were conducted in triplicate and the outcomes are represented as mean value \pm standard deviation. Statistical data were analyzed using a one-way analysis of variance (ANOVA) followed by a post hoc test (Tukey's test) using GraphPad Prism 7, and the $P$ value less than 0.05 is determined as the significance threshold.

\section{Results and Discussion}

3.1. Solubility Studies and Selection of NE Components. For intranasal formulations, it is essential to select safe, nonirritating, and nonsensitizing excipients. Due to limited nasal capacity, the volume of formulation which can deliver the therapeutic dose to the site of action should be minimized as much as possible. So, the solubilizing capacity of the oil phase of NEs is important to reduce this volume [27]. Figure 1 represents the solubility of TMZ in various oils, surfactants, and cosurfactants. According to the graph, the solubilization of TMZ was the highest in triacetin $(4.75 \pm 0.21 \mathrm{mg} / \mathrm{ml})$ as an oil, Transcutol ${ }^{\circledR} \mathrm{P}$ $(8.96 \pm 0.80 \mathrm{mg} / \mathrm{ml})$ as a cosurfactant and labrasol $(12.35 \pm 0.52 \mathrm{mg} / \mathrm{ml})$ as a surfactant in comparison to other substances. 


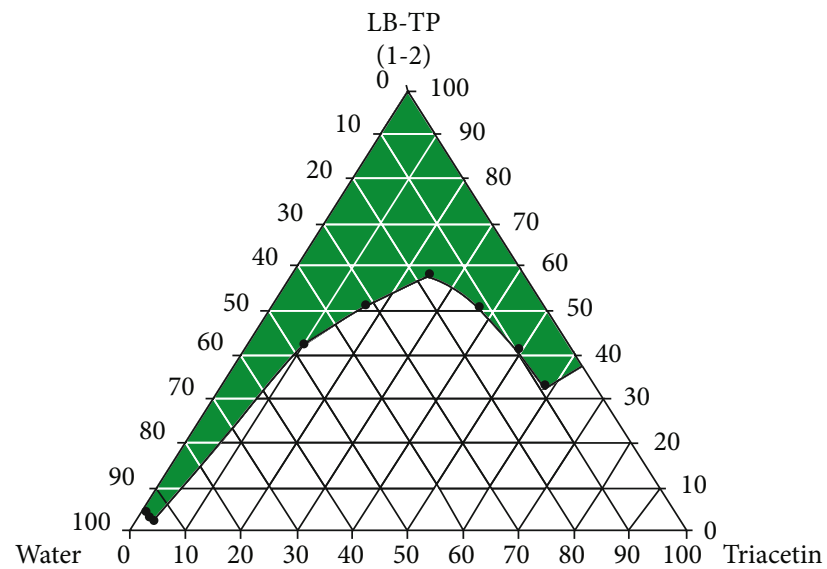

(a)

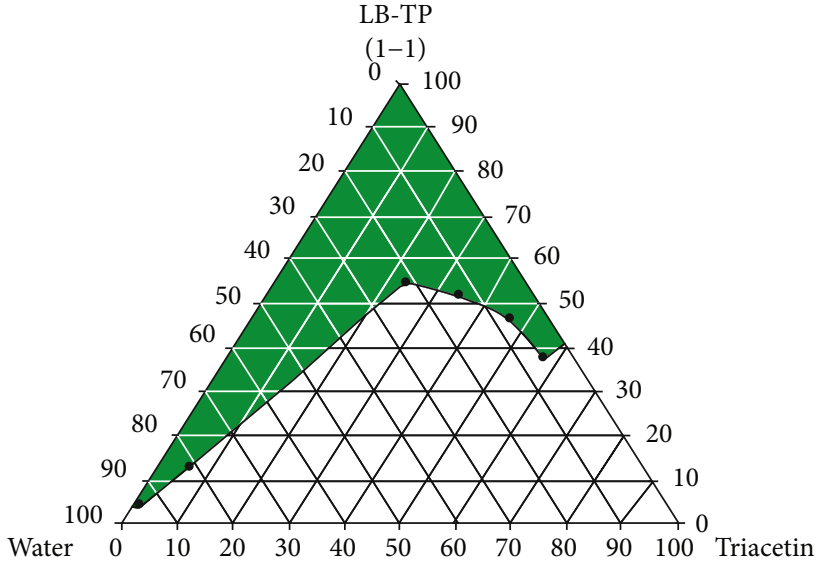

(b)

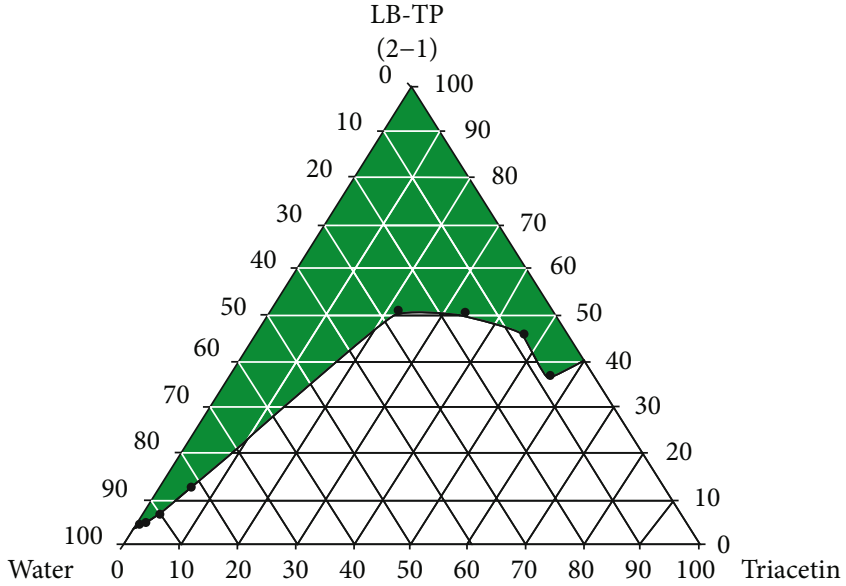

(c)

Figure 2: Partial pseudoternary phase diagram of systems consist of water, oil (Triacetin), mixture of surfactant (Labrasol), and cosurfactant (Transcutol ${ }^{\circledR} \mathrm{P}$ ) with different surfactant: cosurfactant ratio of (a) $1: 2$, (b) $1: 1$, and (c) $2: 1$ at room temperature (Green area of phase diagrams shows the NE region).

In this study, triacetin, a monotriglyceride was selected as the oil phase because of the high capacity for solubilizing of TMZ. Labrasol, a nonionic surfactant with a hydrophiliclipophilic balance (HLB) of 14, was selected as a component due to desirable solubilizing effect. Furthermore, the toxicity of nonionic surfactants is lesser than ionic ones, and they exert lower critical micelle concentration (CMC). They are also compatible with numerous agents used in the formulation of NEs. Their emulsifying properties are not affected by $\mathrm{pH}$ and offer better in vivo stability of o/w NEs [41, 42]. Labrasol is reported to increase the permeability of tight junctions and is being extensively used to improve the absorption of poorly absorbed drugs via different routes, including intranasal, oral, and transdermal $[43,44]$.

To achieve desirable NEs, the use of cosurfactant along with surfactant is necessary to reduce the tension of the interface and provide the interfacial film adequate flexibility which leads to a higher entrapment of drugs [35]. Transcutol ${ }^{\circledR} \mathrm{P}$ (diethylene glycol monoethyl ether) was selected as the cosurfactant based on its solubilizing capacity for TMZ, high biocompatibility, and penetration enhancing properties $[45,46]$.
3.2. Construction of Partial Pseudoternary Phase Diagrams. The partial pseudoternary phase diagrams of NEs consisting of triacetin and three different $S_{\text {mix }}$ (Labrasol and Transcutol ${ }^{\circledR} \mathrm{P}$ with weight ratios $\left.2: 1,1: 1,1: 2\right)$ were constructed by water titration method to determine NE domains. Figure 2 shows these three pseudoternary phase diagrams.

As it can be observed, oil solubilizing capacities enhances with increasing surfactant/cosurfactant content, irrespective of the $S_{\text {mix }}$. This phase study also revealed that the maximum NE region was obtained when surfactant to cosurfactant ratio was $2: 1$.

As the concentration of the labrasol was increasing, higher solubilization of oil occurred. On the other hand, even though it seems the use of cosurfactant (Transcutol ${ }^{\circledR} \mathrm{P}$ ) along with the surfactants leads more stable interfacial film formation and modifies the film curvature but when an extra amount of the cosurfactant presents, it also enters the oil core of NEs, resulting in elevating droplet size and turbid experience [47].

Regarding the result obtained by partial pseudoternary phase diagram construction, the amount of each component for all proposed TMZ NEs was selected from the diagram 
TABLE 1: Composition of NEs containing TMZ.

\begin{tabular}{lcccc}
\hline $\begin{array}{l}\text { Formulation } \\
\text { code }\end{array}$ & $\begin{array}{c}\text { Labrasol } \\
(w / w \%)\end{array}$ & $\begin{array}{c}\text { Transcutol }{ }^{\circledR \mathrm{P}} \\
(w / w \%)\end{array}$ & $\begin{array}{c}\text { Triacetin } \\
(w / w \%)\end{array}$ & $\begin{array}{c}\text { Water } \\
(w / w \%)\end{array}$ \\
\hline NE1 & 13.3 & 6.7 & 2.5 & 77.35 \\
NE2 & 13.3 & 6.7 & 5 & 74.85 \\
NE3 & 20 & 10 & 2.5 & 67.35 \\
NE4 & 20 & 10 & 5 & 64.85 \\
\hline
\end{tabular}

${ }^{*} \mathrm{TMZ}$ was used in $0.15 \% w / w$ concentration in all NE formulations.

with the surfactant to cosurfactant ratio of $(2: 1)$ and enough distance from the boundaries to ensure the stability of the prepared formulations.

3.3. Preparation of NEs Containing TMZ. A low energy method was applied to fabricate TMZ NEs. $0.15 \% \mathrm{w} / \mathrm{w}$ TMZ was incorporated into the oily phase of each $\mathrm{O} / \mathrm{W}$ NEs selected based on pseudoternary phase diagrams (Table 1). All prepared NEs were transparent with any physical instability during $72 \mathrm{~h}$ monitoring at room temperature.

3.4. Physicochemical Characterization of NEs Containing TMZ. The physicochemical properties of prepared NEs were measured (Table 2).

All NEs represented proper droplet size in the range from $19 \mathrm{~nm}$ to $23 \mathrm{~nm}$. Particle size is a critical factor for the intranasal delivery of drugs to the CNS, as depletion in global size (below $100 \mathrm{~nm}$ ) leads to a larger surface area, which enhances the rate of drug absorption through olfactory and trigeminal nerve terminations. This elevated absorption at targeting site is attributed to a lower therapeutic dose of active agent and so fewer side effects [48]. PDI value for all NEs was in the range of 0.18 to 0.25 , which indicates all of them have narrow size distribution and spherical shape of droplets.

Slight positive and/or close to neutral zeta value in the range of $1.02 \mathrm{mV}$ to $1.62 \mathrm{mV}$ for all formulation can be explained by the nonionic nature of all emulsifying components including surfactant and cosurfactant [49].

$\mathrm{pH}$ values of NEs were measured between 3.25 and 3.56, which are in the acceptable range for nasal preparation $(6 \pm 3$ ) [50].

The measured viscosity for all the NEs was in the range of $3.51 \mathrm{cP}$ to $5.87 \mathrm{cP}$. Our results demonstrated that the increase in surfactant concentration is relevant to viscosity value.

Although all NEs showed suitable physicochemical properties for intranasal use, NE1 with fewer excipient content and so less toxicity and also a more desirable size and PDI values selected for further studies.

3.5. Preparation of In Situ Gel NEs. Poloxamer derivatives were used as the gelling and mucoadhesive agents in our study. Poloxamers with amphiphilic properties are widely noticed in the formulation of thermosensitive in situ gels. Among these derivatives, poloxamer 407 is more attractive because of high solubilizing potential, insignificant toxicity, desirable drug-release features, and compatibility with most of the pharmaceutical agents. To optimize the gelling temperature of formulations in the nasal cavity, poloxamer 407 was used with Poloxamer 188 in different ratios (Table 3) [33].

3.6. Determination of Gelation Temperature of In Situ Gel NEs. The gelling temperature is the temperature at which sol to gel phase transition occurs. The general structure of poloxamer consists of a hydrophobic core of polyoxypropylene (PPO) and two hydrophilic segments of polyoxyethylene (POE). POP: POE ratio determines each derivative different specifies, including the critical micelle temperature (CMT), which is a temperature that phase transition from sol to gel occurs regards to polymer concentration. Indeed, hydrophobic portion (PPO) is attributed to the reduction in gelling temperature while the hydrophilic portion (PEO) increases it $[33,51]$.

An appropriate gelation temperature of an in situ gel formulation for intranasal administration should be in the range of $30-34^{\circ} \mathrm{C}$ as the formation of the gel below nasal cavity temperature cause limitation for production and usage. On the other hand, fluid and low viscose formulation between 30 and $34^{\circ} \mathrm{C}$ leads to rapid removal from the absorption region after instillation [52]. NE1c with phase transition at $32.2^{\circ} \mathrm{C}$ represents good phase transition temperature for intranasal use (Table 3).

3.7. Physicochemical Characterization of In Situ Gel Containing TMZ. The physicochemical properties of selected in situ gel NE were measured (Table 2).

In comparison to $\mathrm{NE}$, in situ gel preparation showed a significant reduction in droplet size to $16.25 \pm 0.44 \mathrm{~nm}$, which was expected according to poloxamer 407 emulsifying properties. It has been found poloxamer derivatives have a comparatively broad size distribution of PDI, so a slight rise in PDI value up to $0.35 \pm 0.01$ for in situ gel NE can be justified by innate polydispersity of poloxamers [53].

In situ gel NE showed $4.36 \pm 0.13$ for $\mathrm{pH}$ value. Previous studies demonstrated that the addition of poloxamer 407 increases the $\mathrm{pH}$ of hydrogels $[54,55]$. This observed value is near the ideal $\mathrm{pH}$ range of 4.5 to 6.5 for the nasal delivery, which shows the least irritation at the nasal mucosa surface [56].

An approach of in situ gel preparations for intranasal use is to obtain low viscosity state at room temperature, thereby precise dosing upon administration; on the other hand, increased viscosity in the nasal cavity which prevents nasal mucociliary and prolongs drug resistance time [57]. The use of poloxamer as a viscosity-enhancing agent in formulation raised viscosity to $113.57 \mathrm{cP}$, i.e., approximately 33-fold more than NE with the same $S_{\text {mix }}$ content.

3.8. In Vitro Drug Release Studies. Drug release study is a practical method to evaluate the effectiveness of the formulation throughout investigating the effect of different formulation components on its release characterization [58].

The release graph of the aqueous solution, NE, and in situ gel NE containing TMZ is plotted in Figure 3. After half an hour of the test beginning, approximately the entire amount of TMZ released from the solution, whereas this amount was fewer than $80 \%$ for NE and about $60 \%$ for in situ gel NE. 
TABLE 2: Physicochemical characterization of NEs and selected in situ gel NE (mean \pm SD; $n=3$ ).

\begin{tabular}{lcccrc}
\hline Formulation code & Droplet size $(\mathrm{nm})$ & PDI & Zeta potential $(\mathrm{mV})$ & $\mathrm{pH}$ & Viscosity $(\mathrm{cP})$ \\
\hline NE1 & $19.11 \pm 0.59$ & $0.19 \pm 0.02$ & $1.02 \pm 0.08$ & $3.56 \pm 0.05$ & $3.51 \pm 0.28$ \\
NE2 & $19.90 \pm 0.77$ & $0.18 \pm 0.04$ & $1.07 \pm 0.18$ & $3.43 \pm 0.05$ & $4.31 \pm 0.38$ \\
NE3 & $20.37 \pm 0.95$ & $0.25 \pm 0.03$ & $1.62 \pm 0.61$ & $3.33 \pm 0.10$ & $5.75 \pm 0.26$ \\
NE4 & $22.38 \pm 0.79$ & $0.24 \pm 0.03$ & $1.22 \pm 0.22$ & $3.25 \pm 0.07$ & $5.87 \pm 0.23$ \\
NE1c & $16.25 \pm 0.44$ & $0.35 \pm 0.01$ & $2.38 \pm 0.38$ & $4.36 \pm 0.13$ & $113.57 \pm 1.55$ \\
\hline
\end{tabular}

*TMZ was used in $0.15 \% w / w$ concentration in all NE formulation.

TABLE 3: Formulation composition of in situ gel NEs and their gelation temperature.

\begin{tabular}{lcccccc}
\hline Formulation code & $S_{\text {MIX }} \%$ & Oil \% & P407\% (gelling agent) & P188\% (gelling agent) & Water\% & Gelation temperature $\left(C^{\circ}\right)$ \\
\hline NE1a & 20 & 2.5 & 20 & 0 & 57.35 & $24.4 \pm 0.53$ \\
NE1b & 20 & 2.5 & 19 & 1 & 57.35 & $27.6 \pm 0.91$ \\
NE1c & 20 & 2.5 & 18.5 & 1.5 & 57.35 & $32.2 \pm 1.34$ \\
NE1d & 20 & 2.5 & 18 & 2 & 57.35 & $>40$ \\
\hline
\end{tabular}

*TMZ was used in $0.15 \% w / w$ concentration in all NE formulations.

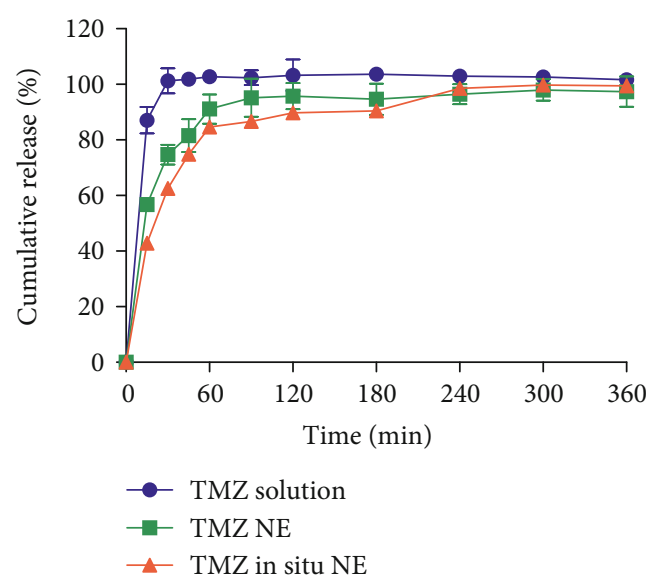

FIGURE 3: Release profile of selected NE and in situ gel NE in comparison to TMZ solution for 6 hours (mean $\pm \mathrm{SD} ; n=3$ ).

Furthermore, the release efficiency of NE and in situ gel NE after $6 \mathrm{~h}$ were $90.78 \pm 3.99 \%$ and $87.65 \pm 0.46 \%$, respectively, clearly lower than the aqueous solution with $99.97 \pm 1.43 \%$ release efficiency $(P<0.01)$. So, it can be concluded that both nanoformulations have sustained release patterns in comparison to the solution.

The reduction in release efficiency for NE and in situ gel NE may be related to the entrapment of TMZ in the oil phase. Drug release from NE involves penetrating it from oil into the interfacial layer and then into the aqueous phase [59]. It seems the addition of poloxamer in in situ gel formulation leads to increased $S_{\text {mix }}$ concentration, which restricts the diffusion of drug to the release medium [60]. Besides, poloxamer derivatives have a core-shell structure, the hydrophobic core as a drug-loading site along with the hydrophilic outer shell influence in the drug release pattern, which can bring about a slow release of the drug [51]. Also, enhancement of viscosity due to the formation of gel could be served as a fur- ther resistant barrier to the drug release from in situ gel NE. This supposition may be boosted by the rheology studies, which revealed that there is an inversely proportional relationship between formulation viscosity and release rate [61].

3.9. Accelerated Physical Stability Studies. Visual observation after 30 minutes centrifuging indicated any instability for both NE and in situ gel NE. Formulations were frozen when they were kept in $-21^{\circ} \mathrm{C}$ during freeze-thaw cycles, but after 30 minutes, keeping at room temperature clarity was recovered. Visual observation also revealed no instability during heating-cooling cycles. The results relating to the size and PDI measurements showed no significant change in these values (Table 4). Therefore, both formulations passed accelerated stability tests.

3.10. Ex Vivo Mucoadhesion Study. The ex vivo mucoadhesion study was performed to estimate the adhesion of developed formulations to the nasal mucosa at the site of absorption. According to the results, the aqueous solution of TMZ as control demonstrated mucoadhesion percentage of $16.1 \pm 2.97$. This percentage has risen to $20.35 \pm 0.58$ for NE. Although this alteration is not statistically significant $(P>0.05)$, but it seems as NEs are more viscous formulations than solutions, they result in elevated residence time of the drug in the nasal cavity [62]. In consequence of adding poloxamer, in situ gel NE showed a noticeable mucoadhesion percentage of $37.037 \pm 2.32(P<0.001)$.

The mucoadhesive effect of Poloxamer 407 and 188 could be clarified by the rheological properties and interaction of these polymers with the mucosa. They have amphiphilic nature which facilitates their interpenetration into the mucus where forming entanglements or noncovalent bonds by the van der Waals and hydrogen forces that result in the adhesion of the polymer to the nasal mucosa $[24,33,63]$.

Besides the adhesion properties of poloxamer, its surfactant nature allows it penetrates into mucous and reaches the epithelia simultaneously [46]. Thanks to mucoadhesive 
TABLE 4: Results relating to droplet size and PDI values after centrifugation, freeze-thaw, and heating-cooling cycles (mean \pm SD; $n=3$ ).

\begin{tabular}{lcccccccc}
\hline & \multicolumn{2}{c}{ Initial values } & \multicolumn{2}{c}{ After freeze-thaw cycles } & \multicolumn{2}{c}{ After heating-cooling cycles } & \multicolumn{2}{c}{ After 30 min centrifugation } \\
Formulations & $\begin{array}{c}\text { Droplet size } \\
(\mathrm{nm})\end{array}$ & PDI & $\begin{array}{c}\text { Droplet size } \\
(\mathrm{nm})\end{array}$ & PDI & $\begin{array}{c}\text { Droplet size } \\
(\mathrm{nm})\end{array}$ & PDI & $\begin{array}{c}\text { Droplet size } \\
(\mathrm{nm})\end{array}$ \\
\hline $\mathrm{NE}$ & $19.11 \pm 0.59$ & $0.19 \pm 0.02$ & $19.58 \pm 0.34$ & $0.19 \pm 0.02$ & $19.86 \pm 0.08$ & $0.19 \pm 0.01$ & $19.76 \pm 0.05$ & $0.18 \pm 0.01$ \\
In situ gel NE & $16.25 \pm 0.44$ & $0.35 \pm 0.01$ & $16.17 \pm 0.55$ & $0.33 \pm 0.04$ & $15.17 \pm 0.43$ & $0.22 \pm 0.05$ & $16.58 \pm 0.32$ & $0.39 \pm 0.02$ \\
\hline
\end{tabular}

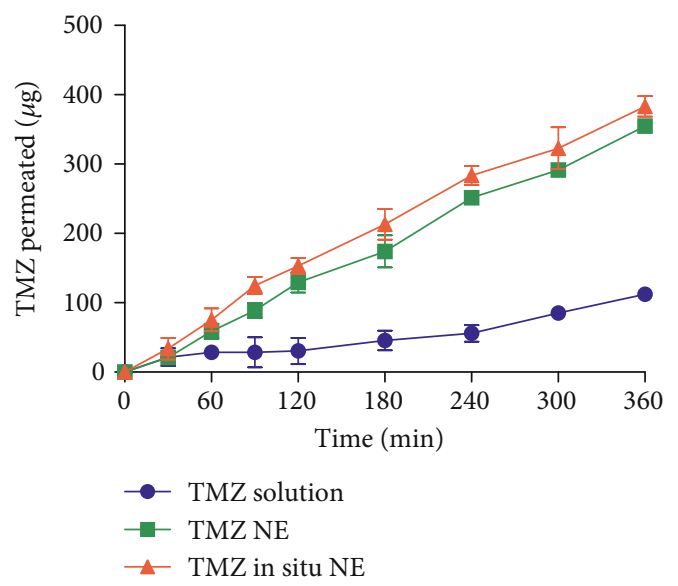

FIgURe 4: Permeation profile of the selected NE and in situ gel NE in comparison to TMZ solution for 6 hours (mean \pm SD; $n=3$ ).

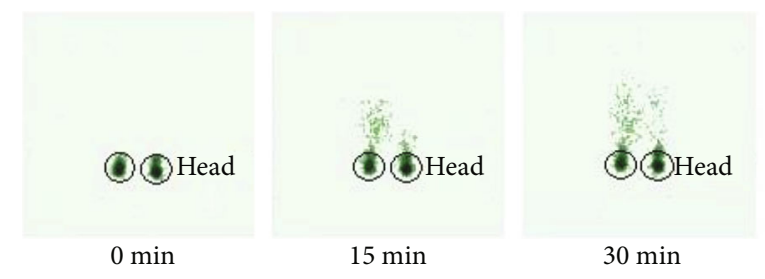

FIGURE 5: Gamma scintigraphy images of TMZ-loaded NE (left side) and in situ gel NE (right side) following intranasal administration at selected time point.

features, poloxamer has been used to develop hydrogels for the direct delivery of many drugs to the brain via nasal route $[64,65]$.

3.11. Ex Vivo Drug Permeation Studies. The permeation profile of the control solution of TMZ, NE, and in situ gel NE through the bovine nasal mucosa has been displayed in Figure 4. The calculated Papp (apparent permeability) of TMZ during $6 \mathrm{~h}$ from in situ gel NE, NE, and control solution were $7.79 \pm 3.68\left(\times 10^{-6} \mathrm{~cm} / \mathrm{s}\right), 7.34 \pm 1.62\left(\times 10^{-6} \mathrm{~cm} / \mathrm{s}\right)$, and $5.12 \pm 2.79\left(\times 10^{-6} \mathrm{~cm} / \mathrm{s}\right)$, respectively. Based on these results, in situ gel NE and NE increased mucosal permeation of TMZ 1.52- and 1.43-fold higher than the control solution, respectively $(P<0.001)$.

The enhanced permeation of the NE formulations in comparison to TMZ solution is related to various characteristics of these systems including nanometric droplet size of the NE, and the presence of $S_{\text {mix }}$ with permeationenhancing properties $[66,67]$.
The size of the nanocarriers is one of the determinative parameters for predicting permeation behavior across the nasal mucosa. It has been reported that colloidal nanoparticles having a size less than $20 \mathrm{~nm}$ can pass on extracellularly to the brain via the intranasal application. The mechanism of endocytosis for intracellular transport to the brain depends on the size of nanocarriers as well. Even though the exact mechanism of transport for nanocarriers via the nasal route is still unknown, both paracellular and transcellular transport of nanosystems to the brain have been distinguished $[68,69]$.

The enhanced permeation for our preparations can be related to $S_{\text {mix }}$ components, i.e., Labrasol and Transcutol ${ }^{\circledR} \mathrm{P}$, regarding their solubilizing potential and penetrating enhancing characteristics [70]. Labrasol also has been identified as the most efficient surfactant in inhibiting the Pglycoprotein (P-gp), which acts as an efflux transporter and so leads to a reduction of the absorption by enterocyte that is a substrate for many lipophilic drugs [58]. Poloxamer might favor intranasal absorption by declining the viscosity and elasticity of the mucus as well as disturbing the lipid membranes and enabling leakage of lipids and proteins from the membranes [71]. As shown in our physicochemical studies, the presence of poloxamer in in situ gel formulation leads to a reduction in droplet size. So, it seems poloxamer with surfactant property increases permeation both by affecting the structure of the mucosa and by reducing the size of the droplets. In similar studies, poloxamer 407 in situ gel formulation of sumatriptan and Labrasol-Transcutol-based mucoadhesive microemulsion loaded with teriflunomide showed an increased permeation rate across the nasal mucosa $[31,52]$.

It can be concluded that the presence of various components in these nanoformulations efficiently enhanced the permeation of TMZ across the nasal mucosa.

3.12. Gamma Scintigraphy. According to the images demonstrated in Figure 5, it could be observed that most part of radiolabeled TMZ in both NE formulations is accumulated in brain upon intranasal administration. A part of radioactivity was detected in gastrointestinal tract after 15 and $30 \mathrm{~min}$ post instillation. As these peripheral distributions are significantly less than the presence of formulations in the brain, it could be concluded that optimized TMZ NE and in situ NE were efficiently uptake to the brain [67].

\section{Conclusion}

In this study, we developed a thermosensitive in situ gel NE with desirable physicochemical properties for the intranasal delivery of TMZ. Using poloxamer, a mucoadhesive gelling 
agent, resistance time at the nasal mucosa surface has been increased. This formulation demonstrated a sustained release pattern and adequate permeation across the nasal mucosa. The gamma scintigraphy studies confirmed the accumulation of NE formulations in the brain. These findings illustrated that in situ gel NE of TMZ could be a promising strategy for the treatment of brain tumors.

\section{Data Availability}

Data are available on request.

\section{Conflicts of Interest}

The authors declare that there is no conflict of interest regarding the publication of this paper.

\section{Acknowledgments}

This research was financially supported by the Hamadan University of Medical Sciences, Hamadan, I.R. Iran (Grant no. 9703221460).

\section{References}

[1] A. Béduneau, P. Saulnier, and J.-P. Benoit, "Active targeting of brain tumors using nanocarriers," Biomaterials, vol. 28, no. 33, pp. 4947-4967, 2007.

[2] L. S. del Burgo, R. M. Hernández, G. Orive, and J. L. Pedraz, "Nanotherapeutic approaches for brain cancer management," Nanomedicine: Nanotechnology, Biology and Medicine, vol. 10, no. 5, pp. e905-e919, 2014.

[3] L. Chu, A. Wang, L. Ni et al., "Nose-to-brain delivery of temozolomide-loaded PLGA nanoparticles functionalized with anti-EPHA3 for glioblastoma targeting," Drug Delivery, vol. 25, no. 1, pp. 1634-1641, 2018.

[4] N. Sanai and M. S. Berger, "Glioma extent of resection and its impact on patient outcome," Neurosurgery, vol. 62, no. 4, pp. 753-766, 2008.

[5] S. Tanaka, D. N. Louis, W. T. Curry, T. T. Batchelor, and J. Dietrich, "Diagnostic and therapeutic avenues for glioblastoma: no longer a dead end?," Nature Reviews Clinical Oncology, vol. 10, no. 1, pp. 14-26, 2013.

[6] J. P. Thakkar, T. A. Dolecek, C. Horbinski et al., "Epidemiologic and molecular prognostic review of glioblastoma," Cancer Epidemiology and Prevention Biomarkers, vol. 23, no. 10, pp. 1985-1996, 2014.

[7] G. Iacob and E. B. Dinca, "Current data and strategy in glioblastoma multiforme," Journal of Medicine and Life, vol. 2, no. 4, pp. 386-393, 2009.

[8] K. Anjum, B. I. Shagufta, S. Q. Abbas et al., "Current status and future therapeutic perspectives of glioblastoma multiforme (GBM) therapy: a review," Biomedicine \& Pharmacotherapy, vol. 92, pp. 681-689, 2017.

[9] J. R. Pineda, M. Jeitany, A. Andrieux, M. P. Junier, H. Chneiweiss, and F. D. Boussin, "Intranasal administration of temozolomide delayed the development of brain tumors initiated by human glioma stem-like cell in nude mice," Journal of Cancer Science \& Therapy, vol. 9, pp. 374-378, 2017.
[10] V. A. Trinh, S. P. Patel, and W. J. Hwu, "The safety of temozolomide in the treatment of malignancies," Expert Opinion on Drug Safety, vol. 8, no. 4, pp. 493-499, 2009.

[11] J. Zhou, K. B. Atsina, B. T. Himes, G. W. Strohbehn, and W. M. Saltzman, "Novel delivery strategies for glioblastoma," The Cancer Journal, vol. 18, no. 1, pp. 89-99, 2012.

[12] M. C. Bonferoni, S. Rossi, G. Sandri et al., "Nanoemulsions for "nose-to-brain" drug delivery," Pharmaceutics, vol. 11, no. 2, p. 84, 2019.

[13] P. G. Upadhaya, S. Pulakkat, and V. B. Patravale, "Nose-tobrain delivery: exploring newer domains for glioblastoma multiforme management," Drug Delivery and Translational Research, vol. 10, no. 4, pp. 1044-1056, 2020.

[14] M. M. Ban, V. R. Chakote, G. N. Dhembre, J. R. Rajguru, and D. A. Joshi, "In-situ gel for nasal drug delivery," International Journal of Development Research, vol. 8, no. 2, pp. 1876318769, 2013.

[15] U. C. Galgatte, A. B. Kumbhar, and P. D. Chaudhari, "Development of in situ gel for nasal delivery: design, optimization, in vitro and in vivo evaluation," Drug Delivery, vol. 21, no. 1, pp. 62-73, 2014.

[16] C. M. Caramella, S. Rossi, F. Ferrari, M. C. Bonferoni, and G. Sandri, "Mucoadhesive and thermogelling systems for vaginal drug delivery," Advanced Drug Delivery Reviews, vol. 92, pp. 39-52, 2015.

[17] C. Karavasili and D. G. Fatouros, "Smart materials: in situ gelforming systems for nasal delivery," Drug Discovery Today, vol. 21, no. 1, pp. 157-166, 2016.

[18] J. Rassing, W. McKenna, S. Bandyopadhyay, and E. Eyring, "Ultrasonic and ${ }^{13} \mathrm{C}-\mathrm{NMR}$ studies on gel formation in aqueous solutions of the ABA block polymer Pluronic RF 127," Journal of Molecular Liquids, vol. 27, no. 3-4, pp. 165-178, 1984.

[19] X. Wang, G. Liu, J. Ma et al., "In situ gel-forming system: an attractive alternative for nasal drug delivery," Critical Reviews $^{T M}$ in Therapeutic Drug Carrier Systems, vol. 30, no. 5, 2013.

[20] G. Dumortier, J. L. Grossiord, F. Agnely, and J. C. Chaumeil, "A review of poloxamer 407 pharmaceutical and pharmacological characteristics," Pharmaceutical Research, vol. 23, no. 12 , pp. 2709-2728, 2006.

[21] L. Mayol, M. Biondi, F. Quaglia et al., "Injectable thermally responsive mucoadhesive gel for sustained protein delivery," Biomacromolecules, vol. 12, no. 1, pp. 28-33, 2011.

[22] S. Mansuri, P. Kesharwani, K. Jain, R. K. Tekade, and N. Jain, "Mucoadhesion: a promising approach in drug delivery system," Reactive and Functional Polymers, vol. 100, pp. 151172, 2016.

[23] Lusiana and C. C. Müller-Goymann, "Preparation, characterization, and in vitro permeation study of terbinafine $\mathrm{HCl}$ in poloxamer 407-based thermogelling formulation for topical application," AAPS PharmSciTech, vol. 12, no. 2, article 9611, pp. 496-506, 2011.

[24] L. Na, S. Mao, J. Wang, and W. Sun, "Comparison of different absorption enhancers on the intranasal absorption of isosorbide dinitrate in rats," International journal of pharmaceutics, vol. 397, no. 1-2, pp. 59-66, 2010.

[25] A. Fisher, M. Watling, A. Smith, and A. Knight, "Pharmacokinetic comparisons of three nasal fentanyl formulations; pectin, chitosan and chitosan-poloxamer 188," International Journal of Clinical Pharmacology and Therapeutics, vol. 48, no. 2, pp. 138-145, 2010. 
[26] A. Khan, M. Aqil, S. S. Imam et al., "Temozolomide loaded nano lipid based chitosan hydrogel for nose to brain delivery: characterization, nasal absorption, histopathology and cell line study," International journal of biological macromolecules, vol. 116, pp. 1260-1267, 2018.

[27] E. M. Abdou, S. M. Kandil, and H. M. El Miniawy, "Brain targeting efficiency of antimigrain drug loaded mucoadhesive intranasal nanoemulsion," International journal of pharmaceutics, vol. 529, no. 1-2, 2017.

[28] Q. Zhang, X. Jiang, W. Jiang, W. Lu, L. Su, and Z. Shi, "Preparation of nimodipine-loaded microemulsion for intranasal delivery and evaluation on the targeting efficiency to the brain," International journal of pharmaceutics, vol. 275, no. 1-2, pp. 85-96, 2004.

[29] R. B. Patel, M. R. Patel, K. K. Bhatt, and B. G. Patel, "Paliperidone-loaded mucoadhesive microemulsion in treatment of schizophrenia: formulation consideration," Journal of Pharmaceutical Innovation, vol. 8, no. 3, pp. 195-204, 2013.

[30] R. Pathak, R. Prasad Dash, M. Misra, and M. Nivsarkar, "Role of mucoadhesive polymers in enhancing delivery of nimodipine microemulsion to brain via intranasal route," Acta Pharmaceutica Sinica B, vol. 4, no. 2, pp. 151-160, 2014.

[31] D. Gadhave, B. Gorain, A. Tagalpallewar, and C. Kokare, "Intranasal teriflunomide microemulsion: an improved chemotherapeutic approach in glioblastoma," Journal of Drug Delivery Science and Technology, vol. 51, pp. 276-289, 2019.

[32] T. Tadros, "Application of rheology for assessment and prediction of the long-term physical stability of emulsions," Advances in colloid and interface science, vol. 108-109, pp. 227-258, 2004.

[33] E. Giuliano, D. Paolino, M. Fresta, and D. Cosco, "Mucosal applications of poloxamer 407-based hydrogels: an overview," Pharmaceutics, vol. 10, no. 3, p. 159, 2018.

[34] H. Ammar, H. Salama, M. Ghorab, and A. Mahmoud, "Development of dorzolamide hydrochloride in situ gel nanoemulsion for ocular delivery," Drug development and industrial pharmacy, vol. 36, no. 11, pp. 1330-1339, 2010.

[35] T. Keck, R. Leiacker, H. Riechelmann, and G. Rettinger, “Temperature profile in the nasal cavity," The Laryngoscope, vol. 110, no. 4, pp. 651-654, 2000.

[36] S. Shafiq and F. Shakeel, "Stability and self-nanoemulsification efficiency of ramipril nanoemulsion containing labrasol and plurol oleique," Clinical Research and Regulatory Affairs, vol. 27, no. 1, pp. 7-12, 2010.

[37] D. Butani, C. Yewale, and A. Misra, "Amphotericin B topical microemulsion: formulation, characterization and evaluation," Colloids and Surfaces B: Biointerfaces, vol. 116, pp. 351-358, 2014.

[38] H. Chen, D. Mou, D. Du et al., "Hydrogel-thickened microemulsion for topical administration of drug molecule at an extremely low concentration," International journal of pharmaceutics, vol. 341, no. 1-2, pp. 78-84, 2007.

[39] S. Harikarnpakdee, V. Lipipun, N. Sutanthavibul, and G. C. Ritthidej, "Spray-dried mucoadhesive microspheres: preparation and transport through nasal cell monolayer," Aaps Pharmscitech, vol. 7, no. 1, pp. E79-E88, 2006.

[40] B. M. Shah, M. Misra, C. J. Shishoo, and H. Padh, "Nose to brain microemulsion-based drug delivery system of rivastigmine: formulation andex-vivocharacterization," Drug delivery, vol. 22, no. 7, pp. 918-930, 2015.

[41] K. Kawakami, T. Yoshikawa, T. Hayashi, Y. Nishihara, and K. Masuda, "Microemulsion formulation for enhanced absorption of poorly soluble drugs: II. In vivo study," Journal of controlled Release, vol. 81, no. 1-2, pp. 75-82, 2002.

[42] A. Azeem, M. Rizwan, F. J. Ahmad et al., "Nanoemulsion components screening and selection: a technical note," Aaps Pharmscitech, vol. 10, no. 1, pp. 69-76, 2009.

[43] C.-X. He, Z.-G. He, and J.-Q. Gao, "Microemulsions as drug delivery systems to improve the solubility and the bioavailability of poorly water-soluble drugs," Expert opinion on drug delivery, vol. 7, no. 4, pp. 445-460, 2010.

[44] H.-M. Piao, P. Balakrishnan, H.-J. Cho et al., "Preparation and evaluation of fexofenadine microemulsions for intranasal delivery," International journal of pharmaceutics, vol. 395, no. 1-2, pp. 309-316, 2010.

[45] S. Mura, M. Manconi, D. Valenti, C. Sinico, A. O. Vila, and A. M. Fadda, "Transcutol containing vesicles for topical delivery of minoxidil," Journal of drug targeting, vol. 19, no. 3, pp. 189-196, 2011.

[46] L. C. Espinoza, M. Silva-Abreu, B. Clares et al., "Formulation strategies to improve nose-to-brain delivery of donepezil," Pharmaceutics, vol. 11, no. 2, p. 64, 2019.

[47] J. Xi, Q. Chang, C. K. Chan et al., "Formulation development and bioavailability evaluation of a self-nanoemulsified drug delivery system of oleanolic acid," AAPS PharmSciTech, vol. 10, no. 1, article 9190, pp. 172-182, 2009.

[48] H. Bshara, R. Osman, S. Mansour, and A. E.-H. A. El-Shamy, "Chitosan and cyclodextrin in intranasal microemulsion for improved brain buspirone hydrochloride pharmacokinetics in rats," Carbohydrate polymers, vol. 99, pp. 297305, 2014.

[49] B. Shah, D. Khunt, M. Misra, and H. Padh, "Non-invasive intranasal delivery of quetiapine fumarate loaded microemulsion for brain targeting: formulation, physicochemical and pharmacokinetic consideration," European Journal of Pharmaceutical Sciences, vol. 91, pp. 196-207, 2016.

[50] T. Adnet, A. C. Groo, C. Picard et al., "Pharmacotechnical development of a nasal drug delivery composite nanosystem intended for Alzheimer's Disease treatment," Pharmaceutics, vol. 12, no. 3, pp. 251-267, 2020.

[51] E. Russo and C. Villa, "Poloxamer hydrogels for biomedical applications," Pharmaceutics, vol. 11, no. 12, p. 671, 2019.

[52] M. M. Omar, N. E. Eleraky, A. M. el Sisi, and O. Ali Hasan, "Development and evaluation of in-situ nasal gel formulations of nanosized transferosomal sumatriptan: design, optimization, in vitro and in vivo evaluation," Drug Design, Development and Therapy, vol. 13, pp. 4413-4430, 2019.

[53] Z. S. Bayindir and U. Badilli, "Preparation of polymeric nanoparticles using different stabilizing agents," Ankara Üniversitesi Eczacılık Fakültesi Dergisi, vol. 38, no. 4, pp. 257-268, 2009.

[54] P. Nnamani, F. Kenechukwu, C. Anugwolu, and A. Attama, "Evaluation of hydrogels based on poloxamer 407 and polyacrylic acids for enhanced topical activity of gentamicin against susceptible infections," Tropical Journal of Pharmaceutical Research, vol. 13, no. 9, pp. 1385-1391, 2014.

[55] K. Małolepsza-Jarmołowska, "The effect of poloxamer 407 on the properties of hydrophilic gels containing lactic acid complexed with chitosan," Progress on Chemistry and Application of Chitin and its Derivatives, vol. 15, pp. 143-148, 2010.

[56] R. Scherließ, "Nasal formulations for drug administration and characterization of nasal preparations in drug delivery," Therapeutic Delivery, vol. 11, no. 3, pp. 183-191, 2020. 
[57] P. Kaur, T. Garg, G. Rath, and A. K. Goyal, "In situ nasal gel drug delivery: a novel approach for brain targeting through the mucosal membrane," Artificial cells, nanomedicine, and biotechnology, vol. 44, no. 4, pp. 1-10, 2016.

[58] V. P. Chavda and D. Shah, "Self-emulsifying delivery systems: one step ahead in improving solubility of poorly soluble drugs," Nanostructures for Cancer Therapy, pp. 653-718, 2017.

[59] W. Sun, X. Ma, X. Wei, and Y. Xu, "Nano composite emulsion for sustained drug release and improved bioavailability," Pharmaceutical research, vol. 31, no. 10, pp. 2774-2783, 2014.

[60] U. Sakulku, O. Nuchuchua, N. Uawongyart, S. Puttipipatkhachorn, A. Soottitantawat, and U. Ruktanonchai, "Characterization and mosquito repellent activity of citronella oil nanoemulsion," International journal of pharmaceutics, vol. 372, no. 1-2, pp. 105-111, 2009.

[61] E. S. Ibrahim, S. Ismail, G. Fetih, O. Shaaban, K. Hassanein, and N. H. Abdellah, "Development and characterization of thermosensitive pluronic-based metronidazole in situ gelling formulations for vaginal application," Acta pharmaceutica, vol. 62, no. 1, pp. 59-70, 2012.

[62] H. Y. Karasulu, Z. E. Şanal, S. Sözer, T. Güneri, and G. Ertan, "Permeation studies of indomethacin from different emulsions for nasal delivery and their possible anti-inflammatory effects," AAPS PharmSciTech, vol. 9, no. 2, pp. 342-348, 2008.

[63] F. Din and G. M. Khan, "Development and characterisation of levosulpiride-loaded suppositories with improved bioavailability in vivo," Pharmaceutical development and technology, vol. 24, no. 1, pp. 63-69, 2019.

[64] V. Sridhar, S. Wairkar, R. Gaud, A. Bajaj, and P. Meshram, "Brain targeted delivery of mucoadhesive thermosensitive nasal gel of selegiline hydrochloride for treatment of Parkinson's disease," Journal of drug targeting, vol. 26, no. 2, pp. 150-161, 2018.

[65] S. Shelke, S. Shahi, S. Jalalpure, and D. Dhamecha, "Poloxamer 407-based intranasal thermoreversible gel of zolmitriptanloaded nanoethosomes: formulation, optimization, evaluation and permeation studies," Journal of liposome research, vol. 26, no. 4, pp. 313-323, 2016.

[66] R. Pangeni, S. Sharma, G. Mustafa, J. Ali, and S. Baboota, "Vitamin E loaded resveratrol nanoemulsion for brain targeting for the treatment of Parkinson's disease by reducing oxidative stress," Nanotechnology, vol. 25, no. 48, article 485102, 2014.

[67] H. S. Mahajan, M. S. Mahajan, P. P. Nerkar, and A. Agrawal, "Nanoemulsion-based intranasal drug delivery system of saquinavir mesylate for brain targeting," Drug delivery, vol. 21, no. 2, pp. 148-154, 2014.

[68] A. R. Khan, M. Liu, M. W. Khan, and G. Zhai, "Progress in brain targeting drug delivery system by nasal route," Journal of Controlled Release, vol. 268, pp. 364-389, 2017.

[69] V. Bourganis, O. Kammona, A. Alexopoulos, and C. Kiparissides, "Recent advances in carrier mediated noseto-brain delivery of pharmaceutics," European Journal of Pharmaceutics and Biopharmaceutics, vol. 128, pp. 337-362, 2018.

[70] S. H. Moghadam, E. Saliaj, S. D. Wettig et al., "Effect of chemical permeation enhancers on stratum corneum barrier lipid organizational structure and interferon alpha permeability," Molecular pharmaceutics, vol. 10, no. 6, pp. 2248-2260, 2013.
[71] D. M. Abouhussein, A. Khattab, N. A. Bayoumi, A. F. Mahmoud, and T. M. Sakr, "Brain targeted rivastigmine mucoadhesive thermosensitive In situ gel: Optimization, in vitro evaluation, radiolabeling, in vivo pharmacokinetics and biodistribution," Journal of Drug Delivery Science and Technology, vol. 43, pp. 129-140, 2018. 